MRI-P-001107

November, 2000

hep-ph/0011375

\title{
Signals of R-parity violating supersymmetry in neutrino scattering at muon storage rings
}

\author{
Anindya Datta円, Raj Gandhi円, Biswarup Mukhopadhyaya [3 \\ Harish-Chandra Research Institute (formerly, Mehta Research Institute), \\ Chhatnag Road, Jhusi, Allahabad - 211 019, India, \\ Poonam Mehta \\ Department of Physics and Astrophysics, University of Delhi, Delhi - 110007
}

\begin{abstract}
Neutrino oscillation signals at muon storage rings can be faked by supersymmetric (SUSY) interactions in an R-parity violating scenario. We investigate the $\tau$-appearance signals for both long-baseline and near-site experiments, and conclude that the latter is of great use in distinguishing between oscillation and SUSY effects. On the other hand, for a wide and phenomenologically consistent choice of parameters, SUSY can cause a manifold increase in the event rate for wrong-sign muons at a long-baseline setting, thereby providing us with signatures of new physics.
\end{abstract}

The increasingly strong empirical indications of neutrino oscillations from the observed solar and atmospheric neutrino deficits [1] have emphasised the need for their independent confirmation in accelerator and reactor experiments. One of the actively discussed possibilities in this connection is a muon storage ring [2, 3] which can act as an intense source of collimated neutrinos impinging upon a fixed target. A $\mu^{-}\left(\mu^{+}\right)$beam can thus produce both $\nu_{\mu}\left(\bar{\nu}_{\mu}\right)$ and $\bar{\nu}_{e}\left(\nu_{e}\right)$, thereby providing an opportunity to test both $\nu_{e}-\nu_{\mu}$ and $\nu_{\mu}-\nu_{\tau}$ oscillations which are the favoured solutions for the two anomalies mentioned above.

In the simplest extensions of the standard model, non-degenerate masses for the different neutrino species (and consequent mixing among them) can account for the oscillation phenomena. Considering, for example, the atmospheric $\nu_{\mu}$ deficit, the SuperKamiokande (SK) results [1] strongly suggest $\nu_{\mu}-\nu_{\tau}$ oscillation with $\Delta m^{2} \simeq 10^{-3}-10^{-2} e V^{2}$ and $\sin ^{2} 2 \theta \simeq 1$. Such oscillation was earlier indicated by the Irving-Michigan-Brookhaven and Kamiokande collaborations, and has been also supported more recently by the SOUDAN-II [4] and MACRO [5] experiments. At a muon storage ring, one therefore expects a certain fraction of the $\nu_{\mu}$ 's to oscillate into $\nu_{\tau}$, depending on the energy and the baseline length. Interaction of these $\nu_{\tau}$ 's

\footnotetext{
${ }^{1}$ E-mail: anindya@mri.ernet.in

${ }^{2}$ E-mail: raj@mri.ernet.in

${ }^{3}$ E-mail: biswarup@mri.ernet.in

${ }^{4}$ E-mail: mpoonam@mri.ernet.in
} 
with the target material will produce $\tau$-leptons, the detection of which may, in the simplest case, be interpreted as additional proof of oscillation [6]. Similarly, the detection of "wrong sign muons' may be a vindication of the $\bar{\nu}_{e}-\bar{\nu}_{\mu}$ oscillation hypothesis, thereby providing one with a probe of the parameter spaces corresponding to the vacuum and matter-enhanced oscillation solutions to the solar neutrino puzzle.

However, the predicted rates of $\tau$-appearance or wrong-sign muons in a given experimental setting can be significantly affected by non-standard interactions. In other words, it is possible for non-oscillation physics to intervene and fake oscillation phenomena. Lepton flavour violation effects in general can mimic the neutrino oscillation signal. Such issues have been already discussed in some detail in a model-independent way in the refs. [7]. For example, it is possible for un-oscillated $\nu_{\mu}$ 's to scatter into $\tau$ 's in an R-parity violating supersymmetric (SUSY) framework (with $R=(-1)^{(3 B+L+2 S)}$ ), by virtue of lepton-number violating trilinear couplings [8]. Also, such couplings can produce $\bar{\nu}_{\mu}$ 's from $\mu^{-}$-decay and thus give rise to $\mu^{+}$'s in the detector even in the absence of oscillation [9]. It is important to know the effects of such interactions for two reasons: (i) to look for enhancement in $\tau$ and wrong-sign muon event rates and thus to uncover SUSY effects, and (ii) to see to what extent the signals supposedly coming from oscillation are faked by such new physics. In this paper, we show that one can answer both questions by combining long-baseline experiments with those in which one places the neutrino detectors at a short distance from the storage ring, where the oscillation probability gets suppressed by the baseline length.

In addition, other non-standard options such as left-right symmetric models and theories with extra gauge bosons also can lead to some of the observable consequences discussed here. However, the rather stringent lower bounds (of the order of $500 \mathrm{GeV}$ and above) on the masses of these bosons suppress the contributions. On the other hand, the very relaxation of lepton number conservation in a SUSY scenario introduces several additional couplings in the theory, not all of which can be excluded with a great degree of severity from currently available experimental results. It is some of these new interaction terms, coupled with the possibility of having sfermions in the mass range of $100-300 \mathrm{GeV}$, that are responsible for the remarkable enhancement of tau-and wrong-sign muon event rates at a neutrino factory. Similarly, lepton number violating couplings also occur in the theories with leptoquarks. In R-parity violating SUSY (with $\lambda^{\prime}$-type couplings), squarks behave in much the same way as scalar leptoquarks. Therefore, the results of our analysis on $\tau$ appearance are equally applicable to the interactions involving scalar leptoquarks with charge $\pm \frac{1}{3}$.

While a long-baseline experiment is of advantage from the viewpoint of oscillation, a detector placed at a near site has also been considered in recent studies [10, 3]. Its merit lies in having a large event rate even when its dimensions are small. In reference [3], for example, $\tau$ detection has been investigated in the context of a detector consisting of an array of tungsten sheets with silicon tracking. Such a detector will be helpful in isolating new $\tau$-producing interactions which can be potential contaminants of the oscillation signature.

Let us first consider $\tau$-appearance through oscillation. For a muon-neutrino with energy $E_{\nu}$ (in $\mathrm{GeV}$ ) traversing a distance $L$ (in $\mathrm{km}$ ), the probability of oscillation into a tau-neutrino is given by

$$
\mathcal{P}_{\nu_{\mu, e} \rightarrow \nu_{\tau}}=\sin ^{2} 2 \theta \sin ^{2}\left[1.27 \Delta m^{2} \frac{L}{E_{\nu}}\right]
$$

where $\Delta m^{2}$ is the mass-squared difference between the corresponding physical states in $e V^{2}$, and $\theta$, the mixing angle between flavours. For a baseline length of, say, $700 \mathrm{~km}$, and a 
muon beam of energy $50 \mathrm{GeV}$, this probability corresponding to the solution space for the atmospheric SK data lies in the range $10^{-3}-10^{-2}$, and is smaller for shorter baselines. In order to obtain the $\tau$-event rate one has to fold the charged current cross-section with this probability as well as the $\nu_{\mu}$ energy distribution, and finally use the effective luminosity appropriate for the cone subtended by the detector, which depends on the area and baseline length.

The presence of scalars carrying lepton $(L)$ or baryon $(B)$ number in a SUSY scenario makes it possible to have one of the above quantum numbers broken while the other is conserved. One can thus avoid undesirable consequences like fast proton decay, and can still be consistent with all other symmetries when R-parity is violated. In such a scenario with broken lepton number, the corresponding part of the superpotential is given (suppressing colour and $S U(2)$ indices) by [9],

$$
W_{\psi}=\epsilon_{i} \hat{L}_{i} \hat{H}_{2}+\lambda_{i j k} \hat{L}_{i} \hat{L}_{j} \hat{E}_{k}^{c}+\lambda_{i j k}^{\prime} \hat{L}_{i} \hat{Q}_{j} \hat{D}_{k}^{c}
$$

where i, j and k are generation indices, $\hat{L}$ and $\hat{Q}$ represent the $\mathrm{SU}(2)$ doublet lepton and quark superfields, and $\hat{E}, \hat{U}$ and $\hat{D}$ denote the right-handed charged lepton, up-type quark and downtype quark superfields respectively. In terms of the component fields (with the sfermion fields characterised by the tilde sign), the trilinear terms above lead to interactions of the form

$$
\begin{aligned}
\mathcal{L}= & \lambda_{i j k}^{\prime}\left[\tilde{d}_{L}^{j} \bar{d}_{R}^{k} \nu_{L}^{i}+\left(\tilde{d}_{R}^{k}\right)^{*}\left(\bar{\nu}_{L}^{i}\right)^{c} d_{L}^{j}+\tilde{\nu}_{L}^{i} \bar{d}_{R}^{k} d_{L}^{j}\right. \\
& \left.-\tilde{e}_{L}^{i} \bar{d}_{R}^{k} u_{L}^{j}-\tilde{u}_{L}^{j} \bar{d}_{R}^{k} e_{L}^{i}-\left(\tilde{d}_{R}^{k}\right)^{*}\left(\bar{e}_{L}^{i}\right)^{c} u_{L}^{j}\right]+h . c . \\
& +\lambda_{i j k}\left[\tilde{e}_{L}^{j} \bar{e}_{R}^{k} \nu_{L}^{i}+\left(\tilde{e}_{R}^{k}\right)^{*}\left(\bar{\nu}_{L}^{i}\right)^{c} e_{L}^{j}+\tilde{\nu}_{L}^{i} \bar{e}_{R}^{k} e_{L}^{j}-(i \leftrightarrow j)\right]+h . c
\end{aligned}
$$

It should be noted that these interaction terms violate both lepton flavour and lepton number. By suitable combinations of two such terms, it is possible to achieve contributions to processes which conserve lepton number but involve transition between different generations. The implications of such interactions have been investigated earlier in the contexts of solar [11] and ultra-high energy neutrinos [12]. At a neutrino factory, they can affect the $\tau$ or wrong sign $\mu$ event rates in the following ways:

1. $\lambda^{\prime}$-type interactions give rise to a $\tau$ starting from a $\nu_{\mu}$ which is produced via standard muon decay.

2. $\lambda$-type interactions produce a $\bar{\nu}_{\mu}$ from muon decay, which subsequently has standard charged-current interaction with the target, leading to a wrong-sign muon.

3. A $\nu_{\tau}$ can be produced as a result of $\lambda$-type interactions in muon decay, which produces a $\tau$ through standard interaction.

4. The $\bar{\nu}_{e}$ from $\mu^{-}$decay may scatter into a $\mu^{+}$via $\lambda^{\prime}$-type interactions with the target.

Here we present results for cases 1 and 2 above. The Feynman diagrams for these two cases are shown in figure 1 , where we have chosen those $\lambda^{\prime}$-and $\lambda$-interactions which make $\tilde{b}$ and $\tilde{\tau}$ the mediators in the corresponding diagrams. Predictions for cases 3 and 4 are qualitatively similar to those for 1 and 2 respectively.

The standard model charged current cross-section for $\nu_{\tau} N \longrightarrow \tau^{-} X$ can be found, for example, in references [3, 6]. In R-parity violating SUSY, a $\nu_{\mu}$ can give rise to the same final 


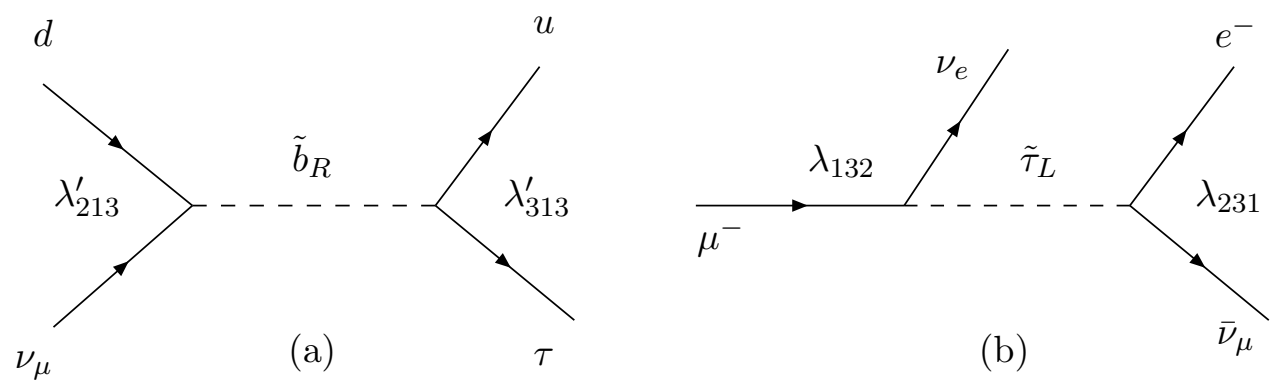

Figure 1: Feynman diagrams for processes producing (a) a $\tau$ or (b) a wrong sign muon in $R$-parity violating SUSY.

state through the couplings $\lambda_{213}^{\prime}$ and $\lambda_{313}^{\prime}$ when the tree-level process is mediated by a b-squark. From considerations of phase-space availability as well as parton densities in a nucleon, the most favourable $\tau$-producing processes at the quark level are $\nu_{\mu} d \longrightarrow \tau^{-} u$ and $\nu_{\mu} \bar{u} \longrightarrow \tau^{-} \bar{d}$. On performing Fierz transformations on the SUSY amplitudes for these processes, one obtains

$$
\begin{aligned}
\mathcal{M}_{S U S Y}\left(\nu_{\mu} d \longrightarrow \tau^{-} u\right) & =\frac{\lambda_{213}^{\prime} \lambda_{313}^{\prime}}{2\left(\hat{s}-m_{\tilde{b}_{R}}^{2}\right)}\left[\bar{u}_{\tau} \gamma_{\mu} P_{L} u_{\nu_{\mu}}\right]\left[\bar{u}_{u} \gamma^{\mu} P_{L} u_{d}\right] \\
\mathcal{M}_{S U S Y}\left(\nu_{\mu} \bar{u} \longrightarrow \tau^{-} \bar{d}\right) & =\frac{\lambda_{213}^{\prime} \lambda_{313}^{\prime}}{2\left(\hat{t}-m_{\tilde{b}_{R}}^{2}\right)}\left[\bar{u}_{\tau} \gamma_{\mu} P_{L} u_{\nu_{\mu}}\right]\left[\bar{v}_{u} \gamma^{\mu} P_{L} v_{d}\right]
\end{aligned}
$$

where $m_{\tilde{b}}$ is the b-squark mass. Left-right mixing in the squark sector has been neglected here. No consequence of the phases of the $\lambda^{\prime}$-type couplings has been considered. It should be noted that the $\left(\nu_{\mu} \leftrightarrow \nu_{\tau}\right)$ oscillation amplitude (arising from neutrino mass splitting) is purely imaginary in a two level analysis 13]. Hence, there is no interference between the oscillation and R-parity violating amplitudes as long as the product of two $\lambda^{\prime}$ couplings is real. We have worked under such an assumption here.

There are phenomenological bounds on the $L$-violating couplings [14]; however, most of these bounds are derived on the assumption that only a single coupling at a time is non-zero (we call them the 'stand-alone' bounds). Thus, although there are individual limits on $\lambda_{213}^{\prime}$ and $\lambda_{313}^{\prime}$, obtained from the universality of charged-current decays of $\pi^{-}$and $\tau$ [14], such limits are not necessarily applicable in the most general case. At any rate, no conclusive limit has been obtained for the product $\left(\lambda_{213}^{\prime} \lambda_{313}^{\prime}\right)$. Hence this product can be treated as a free parameter when it comes to looking for experimental signals. We have also checked that the values of the effective lepton flavour-violating coupling used here do not contradict any limits on such couplings available in the literature.

In figure 2 we show the event rates for tau production for long-baseline experiments, as functions of the baseline length, for a $50 \mathrm{GeV}$ muon beam. When calculating the total $\tau$ appearance rate, we add the R-parity violating contribution with the standard model contribution (from $\nu_{\tau} N \rightarrow \tau^{-} X$ ) after folding the latter with oscillation probability. The results presented here correspond to a sample detector of mass $10 \mathrm{kT}$, with a circular cross-section of $100 \mathrm{~m}^{2}$. Such a specification is similar to that of the ICANOE experiment 15. A muon source producing $10^{20}$ muons per year has been assumed. The expected event rates for both standard charged current and SUSY contributions added to them are displayed. We have used CTEQ4LQ [16] parton distributions to calculate the event rates. The standard model contribution to the $\tau$-production rates has been calculated assuming an oscillation probability 


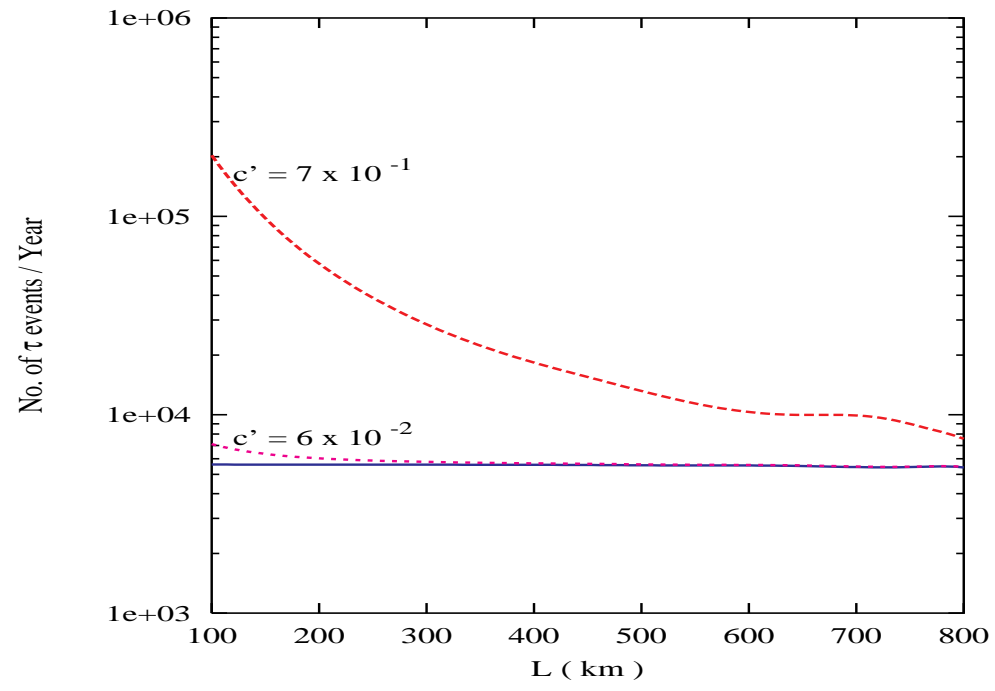

Figure 2: The $\tau$-event rate as a function of the baseline length (L) for $E_{\mu}=50 \mathrm{GeV}$. The solid line corresponds to the contribution from $\nu_{\mu}-\nu_{\tau}$ oscillation, using SK parameters (see text). The two dashed lines refer to cases where the SUSY contributions are included, taking different values of $c^{\prime}\left(\equiv \lambda_{213}^{\prime} \lambda_{313}^{\prime}\right)$.

corresponding to $\Delta m_{23}^{2} \simeq 5.0 \times 10^{-3} \mathrm{eV}^{2}=$ and $\sin ^{2} \theta_{23}=1$, which is within solution space for the atmospheric $\nu_{\mu}$ deficit. An average $\tau$-detection efficiency of $30 \%$ [3, 15] has been used here. Different values of the products of the R-violating couplings have been used, with a bottom-squark mass of $300 \mathrm{GeV}$, which is consistent with current experimental limits. The lower one of these corresponds to the product of the stand-alone bounds of the individual couplings; we also display the results with a value of $c^{\prime}$ which is 10 times greater, and is close to the product of the perturbative limits of the individual couplings. As can be seen from the figure, for baselines of length $\geq 200 \mathrm{~km}$, R-parity violating effects make a serious difference only when the couplings are close to their perturbative limits, while for shorter baselines $(\simeq 100 \mathrm{~km}$ ), they can be competitive even with values on the order of the stand-alone bounds.

However, new physics effects are quite clearly separated when one comes to a near-site detector setting. Here the standard model contribution is suppressed due to the paucity of $\tau$ 's produced in oscillation. In figure 3 we show some plots of $\tau$-event rates with a $1 \mathrm{kT}, 2500 \mathrm{~cm}^{2}$ detector placed at a distance of $40 \mathrm{~m}$ from the storage ring [3]. The two sets of values for the product of the $\lambda^{\prime}$-type couplings already used in the previous figure are also used here; in addition, we show the predictions for two considerably smaller values of this product. One notices a substantial enhancement in the number of $\tau$-events (calculated again with an assumed average detection efficiency of $30 \%$ ) to a level considerably higher than what the standard model predicts. This is the case even when the relevant coupling strengths are much smaller than the limits given in reference [17]. Combining figures 2 and 3, the conclusion, therefore, is that even when the couplings are well within the bounds for the stand-alone situation, near-site effects arising from them lead to overwhelmingly large $\tau$-production, while for long-baseline experiments, contamination of the oscillation signals through R-violating interactions is appreciable when one goes beyond the limits derived on the assumption that only one coupling is non-vanishing at a time.

Similarly, a $\nu_{\tau}$ can also be produced in the decay of the $\mu^{-}$via diagrams of the kind shown in figure 1. It can consequently produce a $\tau$ at the detector even without oscillation. In such a case, the event rates are suppressed by the branching ratio of the R-parity violating decay, 


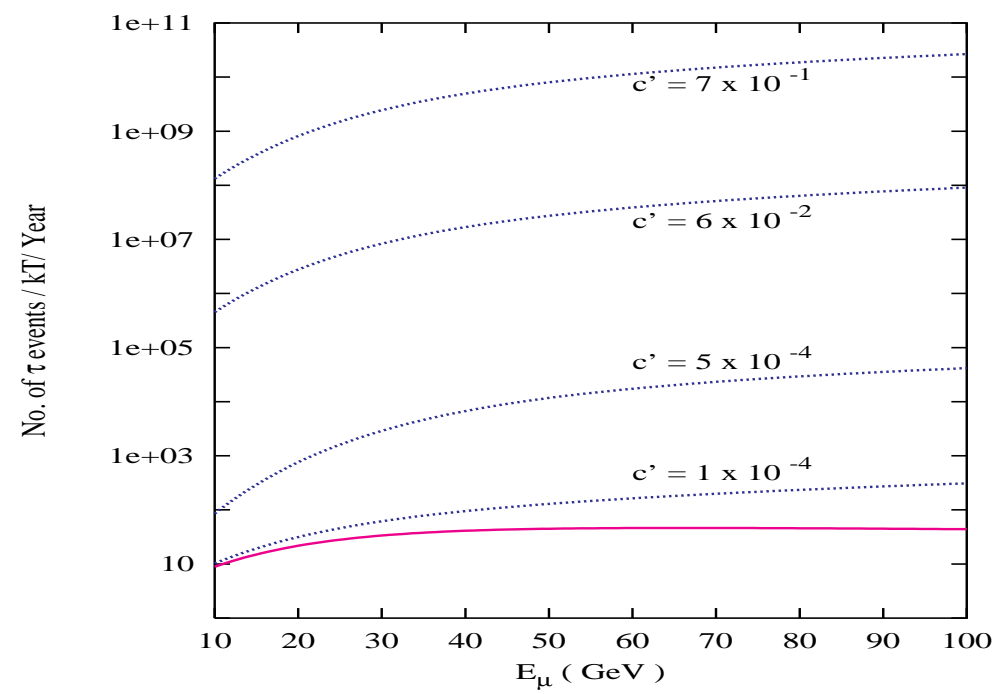

Figure 3: The $\tau$-event rate as function of the muon beam energy for a near-site detector. The solid line shows the oscillation contribution, while the SUSY contributions are included to the dashed lines, using different values of $c^{\prime}\left(\equiv \lambda_{213}^{\prime} \lambda_{313}^{\prime}\right)$.

to an extent depending on the product of the corresponding $\lambda$-couplings. The predictions are similar to the ones discussed above.

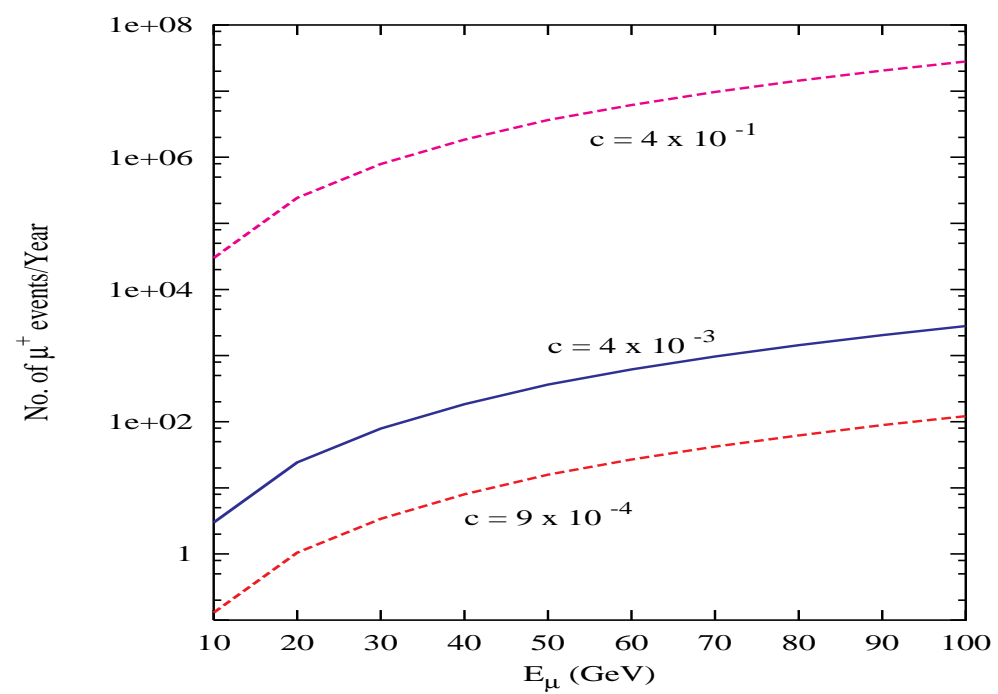

Figure 4: The event rates for wrong-sign muons as functions of muon energy, for a baseline length of $250 \mathrm{~km}$ and a $10 \mathrm{kT}$ detector of area $100 \mathrm{~m}^{2}$. Different values of $c\left(\equiv \lambda_{231} \lambda_{132}\right)$ have been used, with $m_{\tilde{\tau}}=100 \mathrm{GeV}$

Next, we consider wrong-sign muons produced due to R-parity violating effects in muon decays. The Mikhyev-Smirnov-Wolfenstein (MSW) solution to the solar neutrino problem with matter-enhanced $\nu_{e}-\nu_{\mu}$ oscillation requires a mass-splitting of $\simeq 10^{-5} \mathrm{eV}^{2}$ between the mass eigenstates [18]. It has been found earlier [6] that with a muon beam energy of upto $50 \mathrm{GeV}$, and with standard charged current interactions, one can hardly expect to see any events given this kind of mass-splitting, for any realistic baseline length. The situation is even worse for the vacuum oscillation solution which requires $\Delta m^{2} \simeq 10^{-10} \mathrm{eV}^{2}$. Thus a sizable event rate for wrong-sign muons at a long-baseline experiment should be interpreted as a signal of some 


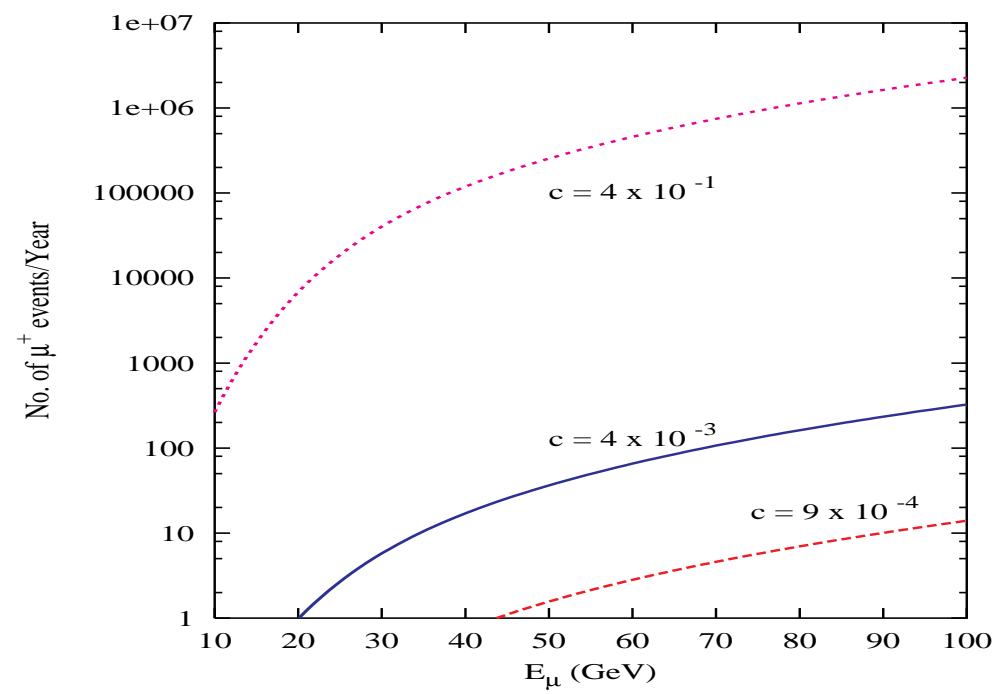

Figure 5: The event rates for wrong-sign muons as functions of muon energy, for a baseline length of $732 \mathrm{~km}$ and a $10 \mathrm{kT}$ detector of area $100 \mathrm{~m}^{2}$. Different values of $c\left(\equiv \lambda_{231} \lambda_{132}\right)$ have been used, with $m_{\tilde{\tau}}=100 \mathrm{GeV}$

new effect, unless $\nu_{e}-\nu_{\mu}$ oscillation is not the solution to the solar neutrino puzzle. In the latter situation, however, the predicted wrong-sign muon rates allow one to probe the solution space to, for example, the LSND results [19]. In such a case, it becomes even more important to understand the potential contributions coming from new physics effects such as R-parity violating SUSY. In the discussion below, we have tried to demonstrate our main point by confining ourselves to solar neutrino solution space, and showing the visibility of the events through SUSY interactions.

Equation (3) tells us that the $\nu_{\mu}$ produced in $\mu$-decays cannot give rise to a $\mu^{+}$through $\mathrm{R}$ parity-violating interactions unless there is substantial left-right mixing in the (D-type) squark sector. On the other hand, diagrams of the kind shown in figure 1(b) can lead to decays like $\mu^{-} \longrightarrow \nu_{\epsilon} e \bar{\nu}_{\mu}$. This decay is governed, for example, by the product $\lambda_{231} \lambda_{132}$ when the process is mediated by a stau. The decay amplitude, as obtained from figure 1 , is

$$
\mathcal{M}_{S U S Y}\left(\mu \longrightarrow \nu_{e} \text { e } \overline{\nu_{\mu}}\right)=\frac{\lambda_{132} \lambda_{231}}{\left(s_{1}-m_{\tilde{\tau}_{L}}^{2}\right)}\left[\bar{u}_{\nu_{e}} P_{R} u_{\mu}\right]\left[\bar{u}_{e} P_{L} u_{\nu_{\mu}}\right]
$$

where $s_{1}=\left(p_{\mu}-p_{\nu_{e}}\right)^{2}$

In figures 4 and 5 we show the event rates for a typical ICANOE-type detector as functions of the muon beam energy, using different values of the above product. Predictions are made for two different baseline lengths, one corresponding to the K2K proposal (figure 4) and the other, to the Fermilab-SOUDAN or CERN-Gran Sasso long baseline experiment. Again, the value 0.004 corresponds to the product of the stand-alone bounds. In addition, two other values, one close to the perturbative limit and the other one considerably smaller, have been used. It may be noted that the only limit on the relevant product c, attempted from the absence of muonium-antimuonium conversion [20], is about $6.3 \times 10^{-3}$. Two of the three values of $\mathrm{c}$ taken here are consistent with these limits. Substantial event rates are produced even with such values. In addition, a general limit on the parameter, $g_{R R}^{s}$ 円denoting the scalar coupling

\footnotetext{
${ }^{1}$ The most general scalar interaction leading to the decay of a muon to a right-handed electron can be written as: $\mathcal{L}=\frac{4 G_{F}}{\sqrt{2}}\left[g_{R R}^{S}\left\langle\bar{e}_{R}\left|\Gamma^{S}\right|\left(\nu_{e}\right)_{L}\right\rangle\left\langle\left(\bar{\nu}_{\mu}\right)_{L}\left|\Gamma^{S}\right| \mu_{R}\right\rangle\right]$. The current experimental limit on $g_{R R}^{S}$ constrains it to be
} 
of a muon leading to its decay into a right-handed electron exists in the literature. Such a limit translates to $c<0.022$, with which a large part of the parameter space covered in the figures is consistent.

Even with conservative choices of the interaction strengths, a clear prediction of ten to several hundred events can be observed for $E_{\mu} \simeq 50 \mathrm{GeV}$, in the R-parity violating case, while no events are expected so long as the masses and mixing in the $\nu_{\mu}-\nu_{\epsilon}$ sector offer a solution to the solar deficit. Clearly, a shorter baseline such as the one shown in figure 4 is of greater advantage. Furthermore, a near-site detector should be able to detect a huge abundance of events, although backgrounds caused by muons produced upstream of the detector pose additional problems there. Taking everything into account, we conclude that that a far-site detector with modest baseline-length like the one studied in figure 4 is probably the optimal answer to questions on possible contributions to wrong-sign muon signals. This statement, however, ceases to be valid if the mass-squared splitting corresponding to $\nu_{e}-\nu_{\mu}$ oscillation belongs, for example, to the solution space for the LSND results. A confirmation (or otherwise) of the LSND claim is expected to come from the MiniBooNE experiment. In case of a reaffirmation of such kind of $\nu_{e}-\nu_{\mu}$ oscillation, for which substantial wrong-sign muon rates are predicted in long-baseline experiments, one has to worry about possible faking by SUSY processes discussed here. Under such circumstances, one has to combine the observed data with those obtained from a near-site detector to separate the two types of effects.

In conclusion, we have investigated the effects of the R-parity violating trilinear couplings on the suggested signals of neutrino oscillations at a muon storage ring. We find that, while the new couplings have to be on the higher side to show a detectable enhancement in the $\tau$-appearance rate with long baselines, even tiny R-violating couplings can lead to very large number of $\tau$ 's at a near-site detector, much in excess of what is expected via oscillation. Nearsite experiments can thus be recommended for isolating new physics effects that fake signals of neutrino oscillation. On the other hand, a class of R-violating interactions, with strengths well within their current experimental limits, can be responsible for an enhanced rate of wrong sign muons at a long-baseline experiment. Since the solution space for the solar neutrino puzzle does not permit such event rates, such muons, if observed at a neutrino factory, can therefore be greeted as harbingers of some new physics, of which R-parity violating SUSY is a favoured example.

Acknowledgement: We thank Sukanta Dutta for help at the initial stage of this work. P.M. acknowledges financial support from the Council for Scientific and Industrial Research, India, and the hospitality of Harish-Chandra Research Institute where this work has been done.

\section{References}

[1] Y. Suzuki, talk given at the XIX International Conference on Neutrino Physics \& Astrophysics, see, http://nu2000.snd. laurentian.ca/Y. Suzuki; H. Sobel, talk given at the same conference, see, http://nu2000.sno.laurentian.ca/H.Sobe

[2] S. Geer, Phys. Rev. D57, 6989 (1998); D. Ayres et al., (electronic archive: physics/9911009); A. Blondel et al., CERN-EP-2000-05.

[3] C. Quigg, (hep-ph/9803326); C. Albright et al. (hep-ex/0008064); S. Geer, (hep$\mathrm{ph} / 0008155)$.

less than 0.066 [21]. 
[4] T. Mann, talk given at the XIX International Conference on Neutrino Physics \& Astrophysics, see, http://nu2000.snd. laurentian.ca/T. Mann.

[5] B. Barish, talk given at the XIX International Conference on Neutrino Physics \& Astrophysics, see, http://nu2000.snd. laurentian.ca/B. Barish.

[6] A. De Rujula, M.B. Gavela, P. Hernandez, Nucl. Phys. B547, 21 (1999); S. Dutta, R. Gandhi, B. Mukhopadhyaya, (hep-ph/9905475), to be published in Euro. Phys. J C; V. Barger, S. Geer, R. Raja, K. Whisnant, Phys. Lett. B485, 379 (2000); Phys. Rev. D62, 013004 (2000); I. Mocioiu and Robert Shrock, Phys. Rev. D62, 053017 (2000).

[7] S. Bergmann, Y. Grossman, D. Pierce, Phys. Rev. D61, 053005 (2000); L. Jhonson, D. Mckay, Phys. Rev. D61, 113007 (2000); S.Bergmann et al., Phys. Rev. D62, 073001 (2000); S. Bergmann, H. Klapdor-Kleingrothaus, H. Pass, Phys. Rev. D62, 113002 (2000).

[8] P. Fayet, Phys. Lett. B69, 489 (1977); G. Farrar and P. Fayet, Phys. Lett. B76, 575 (1978).

[9] V. Barger, G.F. Giudice, and T. Han, Phys. Rev. D40, 2987 (1989); also see the second reference of [3].

[10] V. Barger, S. Geer, R. Raja, K. Whisnant, (hep-ph/0007181).

[11] V. Barger, R. Phillips, K. Whisnant, Phys. Rev. D44, 1629 (1991).

[12] M. Carena et al., Phys. Rev. D58, 095003 (1998).

[13] P. B. Pal, Int. J. Mod. Phys. A7, 5387 (1992).

[14] R. Barbier et al., (hep-ph/9810232) and references therein; C. Allanach, A. Dedes and H. Dreiner, Phys. Rev. D60, 075014 (1999).

[15] ICANOE Proposal, LNGS-P21/99, see, http://pcnometh4.cern.ch; A. Rubbia, talk given at International Conference on Next Generation Nucleon Decay and Neutrino Detector (NNN '99), (hep-ex/0001052).

[16] H. Lai et al., Phys. Rev. D55, 1280 (1997).

[17] See the last reference of [14].

[18] J. J. Bahcall, P. I. Krastev, A. Yu. Smirnov, Phys. Rev. D58, 096016 (1998); Phys. Rev. D60, 093991 (1999); M. Gonzalez-Garcia, Talk given at the XIX International Conference on Neutrino Physics \& Astrophysics, see, http://nu2000.sno.laurentian.ca/M. GonzalezGarcia; J. Bachall, P. Krastev and A. Smirnov, (hep-ex/0103179).

[19] LSND Collab., C. Athanassopoulos et al., Phys. Rev. Lett. 75, 2650 (1995); ibid. 77, 3082 (1996); Phys. Rev. C58, 2489 (1998).

[20] J E. Kim, P. Ko, D. Lee, Phys. Rev. D56, 100 (1997).

[21] D. E. Groom et al., Euor. Phys. J. C15, 1 (2000). 